\title{
Fast Method for Calculating Spatially Resolved Heterogeneous Electron-Transfer Kinetics and Its Application to Graphene with Defects
}

\author{
Pavlov S.V. ${ }^{1,2}$, Kislenko V.A. ${ }^{1}$, Kislenko S.A. ${ }^{{ }^{*}}$ \\ ${ }^{1}$ Joint Institute for High Temperatures, Izhorskaya 13/2, Moscow, 125412, Russian Federation \\ ${ }^{2}$ Skolkovo Institute of Science and Technology, Nobel Str. 3, Moscow, 143026, Russian \\ Federation \\ *kislenko@ihed.ras.ru
}

\section{S1. Acceptor orbitals of redox species}

To obtain the final wave functions of the electrons transferred from the electrode surface to redox species, we have calculated the electronic structure of the following molecules in the reduced states: $\mathrm{O}_{2}^{-}, \mathrm{IrCl}_{6}^{3-}, \mathrm{Ru}\left(\mathrm{NH}_{3}\right)_{6}^{2+}$ We use density functional theory (DFT) calculations with b3lyp exchange correlation functional and lanl2dz basis set. Figures S1-S3 shows isosurfaces of the corresponding highest occupied molecular orbitals (wave functions of the final state). 

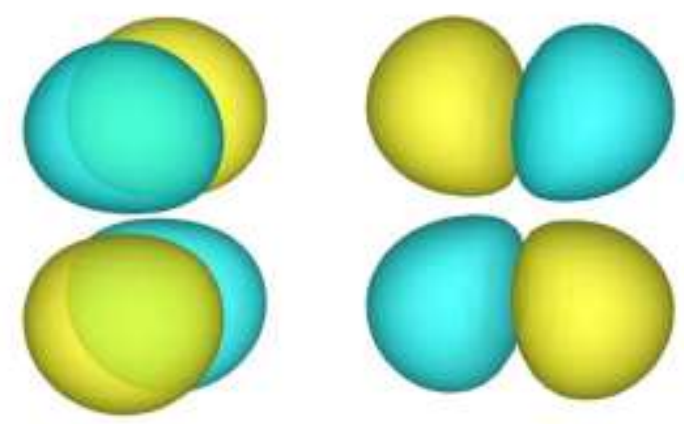

Figure S1. The highest occupied molecular orbitals (HOMO) of $\mathrm{O}_{2}^{-}$
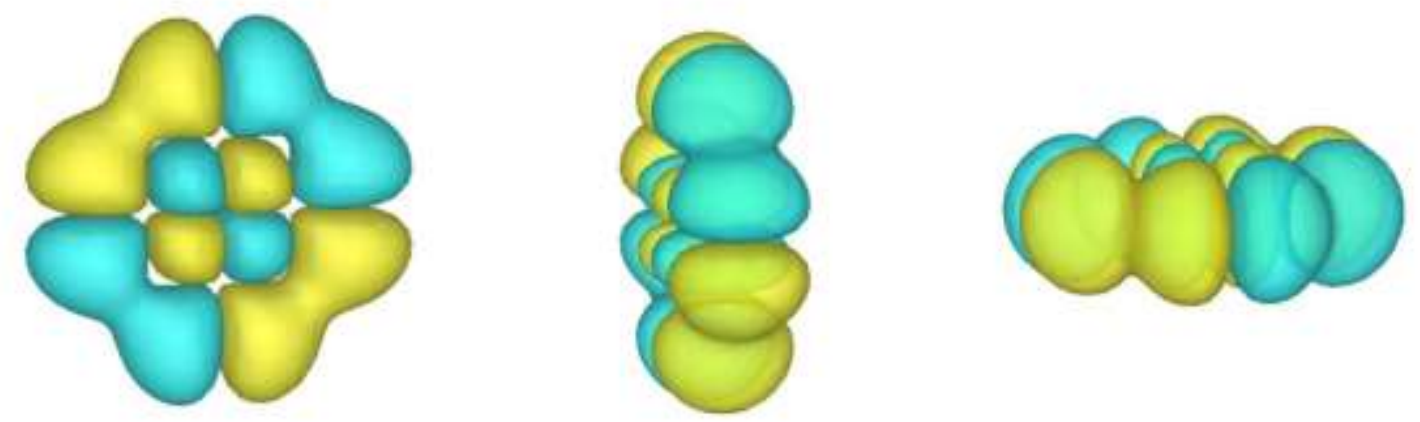

Figure S2. The highest occupied molecular orbitals (HOMO) of $\operatorname{IrCl}_{6}^{3-}$
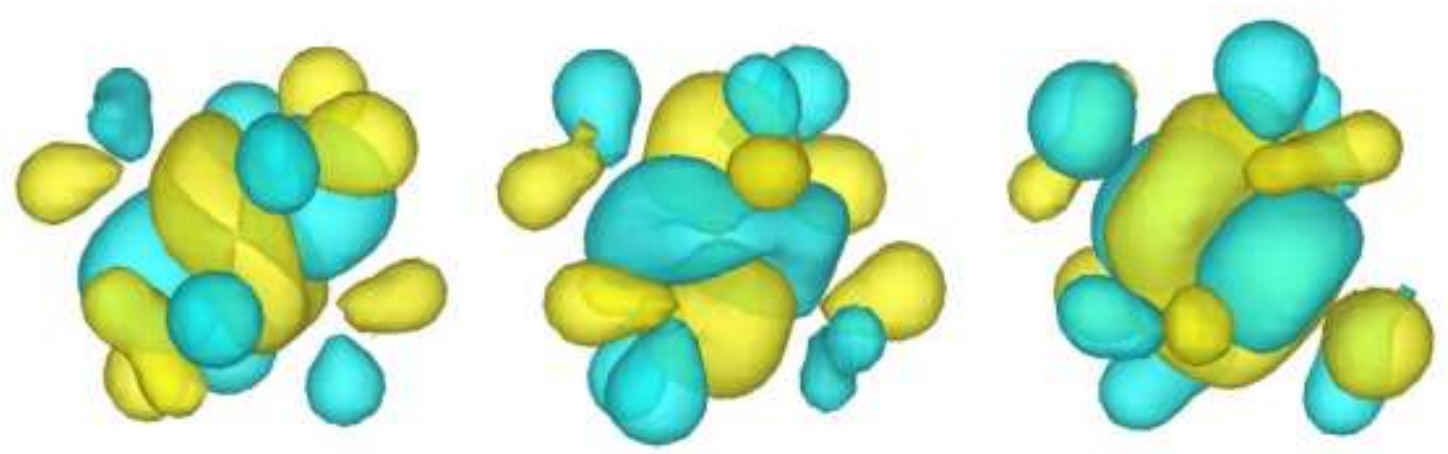

Figure S3. The highest occupied molecular orbitals (HOMO) of $\mathrm{Ru}\left(\mathrm{NH}_{3}\right)_{6}^{2+}$

\section{S2. Technical details}

After we obtain the electronic structure of the studied surfaces, the following procedure of data post-processing was used:

1) Obtain the electronic wave functions $\psi_{i}(\varepsilon)$ of the studied surface on the $3 \mathrm{D}$ grid for each energy value $\varepsilon$ using the module vaspwfc (https://github.com/QijingZheng/VaspBandUnfolding) 
- in this particular study we use integration limits from $-4 \mathrm{eV}$ to $7 \mathrm{eV}$ with the energy step of $0.01 \mathrm{eV}$, the step of the grid was $0.5 \mathrm{Bohr}$ in all coordinate directions;

2) Calculate (up to a constant) the electronic coupling matrix elements $H_{i f}(\varepsilon, \vec{r})$ for each energy $\varepsilon$ and redox molecule position $\vec{r}$ using: (2a) - overlap integral with the corresponding orbital of a redox molecule; (2b) - analytical Tersoff-Hamann or Chen approximations;

2a) Generate the wave function of the final state of the electron $\psi_{f}$ on the same grid from the GTO DFT calculation of the isolated redox molecule in the reduced state. In practice $\psi_{f}$ can be degenerate, for instance, doubly in case of $\mathrm{O}_{2}{ }^{0 /-}$ and triply in case of $\mathrm{Ru}\left(\mathrm{NH}_{3}\right)_{6}{ }^{3+/ 2+}$ and $\mathrm{IrCl}_{6}{ }^{2-/ 3-}$ (see figure S1-S3). Therefore, one should take into account those overlap integral that gives the maximum value for each position $\vec{r}$ :

$$
S_{i f}(\varepsilon, \vec{r})=\max _{(d)}\left[\int_{\Omega} \psi_{i}(\varepsilon) \psi_{f}^{(d)}(\vec{r}) d \Omega\right]
$$

where $(d)$ is the index of degenerate states;

2b) Calculate the electronic coupling matrix elements using the wave function $\psi_{i}(\varepsilon, \vec{r})$ or corresponding derivatives according to equations (6a-6b);

3) Set the parameters: the reorganization energy $\lambda$, the double-layer capacitance $C_{E D L}$, the standard potential of a redox couple $V^{0}$, a temperature $T$. Calculate the Fermi level $\varepsilon_{F}^{v a c}$ and estimate the relative positions of the energy distribution of the oxidized states of a redox couple in solvent $W_{o x}$ and the surface density of states $\rho(\varepsilon)$ after equilibration from equation (4).

4) Calculate the rate constant of the electron transfer using the equation (3). If one needs to obtain an absolute value of the rate constant, the proportionality coefficient between matrix element and overlap integral have to be calibrated using one of the accurate and computationally costlier methods of calculating electronic coupling for some sample of positions $\vec{r}$.

The above procedure was implemented using python3 and publicly available on GitHub (https://github.com/vitalyanich/electrochemistry).

The key step in calculating electron transfer rate constant is the estimation (up to a constant) the electronic coupling matrix element. Technically, we obtain effective wave function of the initial state of electron for each energy range from $\varepsilon$ to $\varepsilon+\Delta \varepsilon$ ( $\Delta \varepsilon$ was $0.01 \mathrm{eV}$ in this particular case). Example of the wave function is depicted in figure S4. The figure shows isosurfaces for: a) real and b) imaginary part of the wave function. 

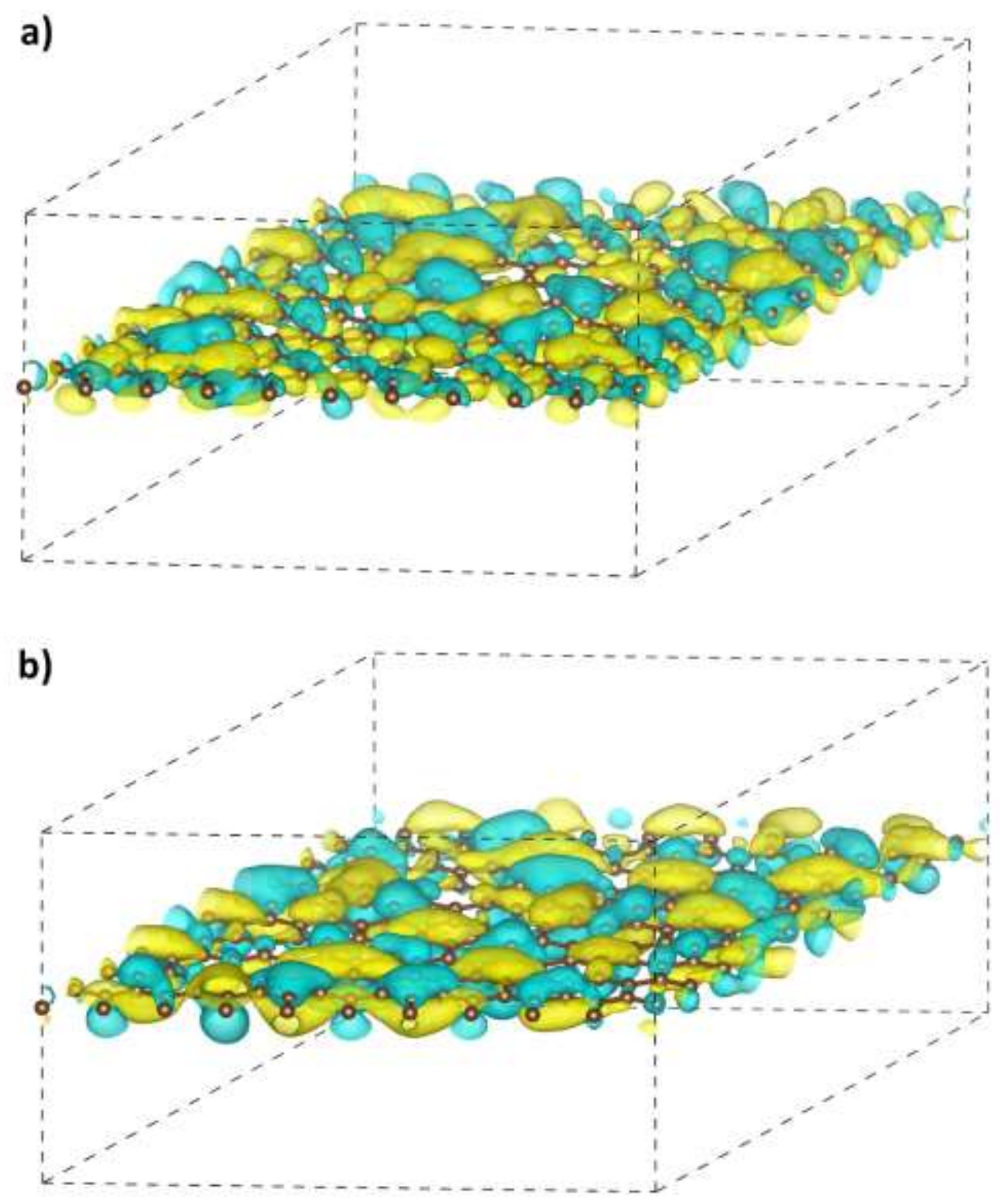

Figure S4. Example of isosurfaces of the wave function of an electron in the graphene surface on grid: a) real part; b) imaginary part.

On the same grid we generate necessary final wave functions from the expansion coefficients to the basis of atomic orbitals, which is approximated as gaussian type orbitals particularly in lanl $2 \mathrm{dz}$ basis set, which was used in this case. An example of the generated final state wave function is shown in figure S5. 


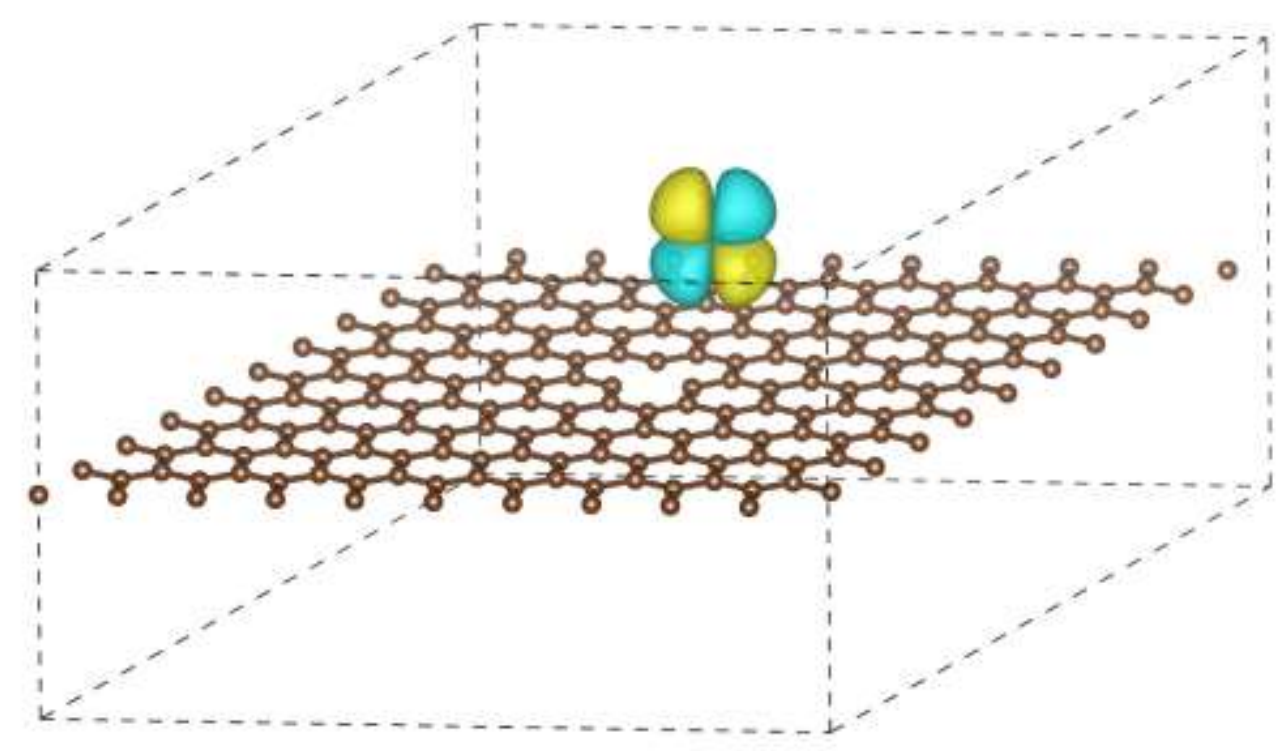

Figure S5. Example of the $\mathrm{O}_{2}$ acceptor wave function generated on grid.

Finally, we calculate the overlap integral of initial and final state numerically on the grid, also we use cutoff distance around point $\vec{r}$ to speed up calculations.

\section{S3. Computational efficiency}

Here we compare the calculation speed for different levels of final wave function description. Figure S6 shows the time needed to calculate the constant-height map of the electron transfer rate constant depending on the type of acceptor orbital. In addition to affecting the complexity of calculations of different numbers of atomic orbitals in the molecular orbital of a redox specie, different cutoff radii were used because of different size of redox molecules: $5 \AA$ for $O_{2}, 6 \AA$ for $\mathrm{IrCl}_{6}$, and $8 \AA$ for $\mathrm{Ru}\left(\mathrm{NH}_{3}\right)_{6}$. As can be seen from figure $\mathrm{S} 6$, analytical approximations of acceptor orbitals (s or $p_{z}$ ) speed up calculations up to 2-3 orders of magnitude. 


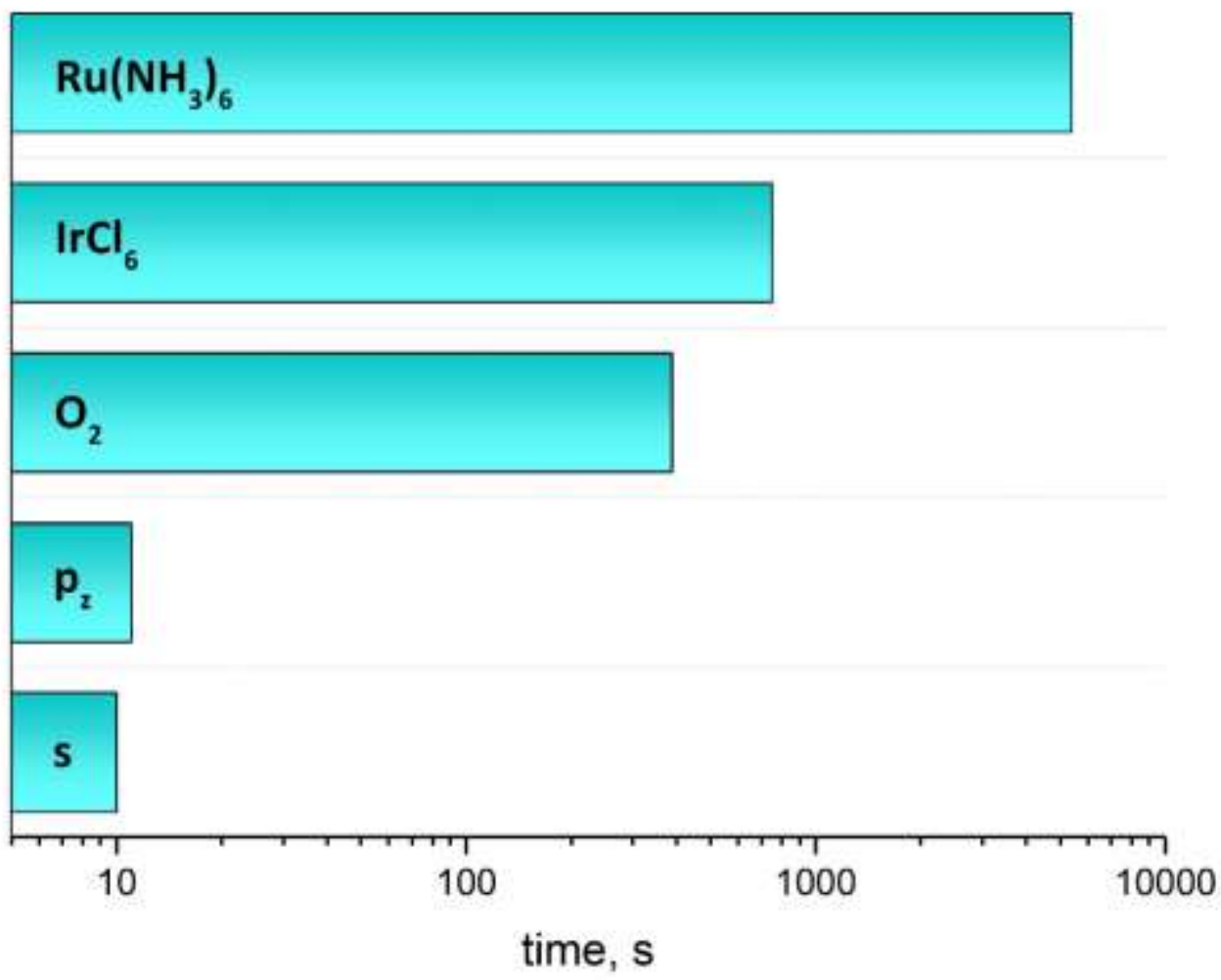

Figure S6. Program run time with different levels of description of the acceptor orbital

\section{S4. Non-linearized form of electronic transmission coefficient}

The rate constant of heterogeneous electron transfer could be expressed as:

$$
k(\vec{r})=\int_{-\infty}^{\infty} \kappa(\varepsilon, \vec{r}) \frac{\omega}{2 \pi} \exp \left(-\frac{\left(\varepsilon-\varepsilon_{F}-\lambda\right)^{2}}{4 k_{B} T \lambda}\right) f(\varepsilon) \rho(\varepsilon) d \varepsilon .
$$

In this work, we used the linearized expression for the electronic transmission coefficient that is applicable for the non-adiabatic electron transfer $\kappa(\varepsilon, \vec{r})$. It is possible to use more general expression of this quantity:

$$
\kappa(\varepsilon, \vec{r})=1-\exp (-2 \pi \gamma(\varepsilon, \vec{r}))
$$

where $\gamma(\varepsilon, \vec{r})$ is the Landau-Zener factor, which can be calculated as follows:

$$
\gamma(\varepsilon, \vec{r})=\frac{2\left|H_{i f}(\varepsilon, \vec{r})\right|^{2}}{\hbar \omega} \sqrt{\frac{\pi}{\lambda k T}}
$$

However, to correctly calculate the rate constant in the transition regime between the nonadiabatic and adiabatic limits, it is necessary to calculate the exact values of electronic coupling parameters $\left|H_{i f}(\varepsilon, \vec{r})\right|$. 